\title{
El descenso de peso durante la lactancia no parece afectar el crecimiento de los hijos
}

The effect of weight loss in overweight, lactating women on the growth of their infants. Lovelady CA, Garner KE, Moreno KL and Williams JP. N Engl J Med 2000; 342: 449-453.

\section{Objetivo}

Evaluar si la pérdida de peso de 0,5 a 1 kilo por semana durante la lactancia en mujeres con sobrepeso afecta el crecimiento de sus hijos.

\section{Diseño}

Ensayo clínico aleatorizado de diez semanas de duración.

\section{Lugar}

Departamento de nutrición de la Universidad de Carolina del Norte, Greensboro, EE.UU.

\section{Pacientes}

Se incluyeron 47 mujeres sanas, sedentarias, no fumadoras, que alimentaban a sus hijos con lactancia exclusiva y que tenían sobrepeso. Su índice de masa corporal (IMC) a las cuatro semanas del postparto estaba entre 25 y $30 \mathrm{~kg} / \mathrm{m} 2$.

\section{Intervención}

Se incorporó a las pacientes en forma aleatorizada a un programa de alimentación durante diez semanas. El plan de alimentación consistía en: a) reducir $500 \mathrm{kcal}$ del valor surgido de promediar la ingestión basal energética promedio y la estimación de la energía requerida (calculando las necesidades debidas a la lactancia) y b) realizar ejercicio aeróbico cuatro veces por semana. El grupo control mantuvo una dieta normal con actividad física una vez por semana. Todas las mujeres recibieron un suplemento polivitamínico con el $50 \%$ de los requerimientos de una mujer que está amamantando.

\section{Resultados}

Un total de 48 mujeres entraron al estudio (27 en el grupo dieta más ejercicio y 21 en el grupo control). Durante las diez semanas del estudio se perdieron seis mujeres en el grupo de estudio y dos en el grupo control, que fueron las más sanas, las que tenían un mayor IMC, los hijos más robustos y el peor nivel de estado físico. No hubo diferencias de crecimiento (peso y altura) en los hijos de las madres de ambos grupos. La ganancia de peso en el grupo intervención fue de $1925 \pm$ $500 \mathrm{~kg}$ y en el grupo control, $1861 \pm 576$. Respecto de la altura, la ganancia en el grupo estudiado fue de $7,8 \pm 2 \mathrm{~cm}$ y en el grupo control, de $7,3 \pm 1,7 \mathrm{~cm}$. La diferencia de los cambios de peso entre los grupos de los niños no fue significativa: $64 \mathrm{~g}$ (IC95\%: $-280 \mathrm{a}+409$ ).

Durante el estudio las mujeres del grupo intervención perdieron peso y espesor de la piel en forma estadísticamente significativa. Al final del estudio, 8 de las 21 mujeres del grupo intervención tenían un IMC menor de 25 (38\%), comparado con 2 mujeres del las 19 del grupo control (11\%).

En el grupo intervención, el $48 \%$ de las mujeres estaba a $1 \mathrm{~kg}$ de su peso previo al embarazo ỳ solo el $21 \%$ en el grupo control. Todas las mujeres del grupo en estudio mejoraron su estado cardiovascular.

\section{Conclusiones}

Una moderada pérdida de peso (de 0,5 kg. por semana) en un programa de restricción dietética y ejercicio programado no afecta el crecimiento de los hijos alimentados con lactancia exclusiva.

\section{COMENTARIO}

El estudio es interesante porque el embarazo es una causa importante de sobrepeso ${ }^{1}$. Frecuentemente nuestras pacientes puérperas solicitan información acerca de la posibilidad de hacer ejercicio y hacer dieta. Tradicionalmente no se recomienda hacer dieta a las mujeres en esta etapa de la vida. ${ }^{2}$ La evidencia previa para esta recomendación es escasa.

Al ser un estudio aleatorizado ambos grupos fueron similares. Hubo una pérdida de 8 pacientes ( 2 en el grupo control y 6 en el grupo intervención) las cuáles eran más sanas, tenían un mayor sobrepeso y menos entrenamiento para el ejercicio.

El principal problema es el número escaso de pacientes incluídas en el estudio, que determina un poder limitado para detectar diferencias de peso entre los niños de ambos grupos; dando un intervalo de confianza muy amplio (gran dispersión de los valores obtenidos). Aunque el peso de los hijos cuyas madres realizaron dieta fue similar a los del otro grupo, la imprecisión de esta estimación hace posible un resultado distinto (en el peor escenario, los hijos de madres a dieta podrían bajar hasta $280 \mathrm{~g}$. más que los del grupo control). Observando las tablas de crecimiento, esta posible pérdida de $280 \mathrm{~g}$ no pondría en riesgo la salud del niño (siempre que no tenga riesgo de desnutrición); pero tal vez el chico pueda bajar de percentilo. ${ }^{3}$
La editorial de la revista sugiere realizar un análisis más refinado, donde se relacione descenso de peso de la madre y peso de los niños. Tampoco sabemos ciertas características de los niños en ambos grupos como por ejemplo, si además de la lactancia tomaron alimentación extra, si los niños del grupo intervención eran más sanos y tampoco se menciona la frecuencia y duración de la alimentación. Un punto importante del estudio radica en que un $50 \%$ de las mujeres llegaron al peso previo al embarazo y gran parte lograron un IMC debajo de 25, sin embargo no se evaluó el estrés, la ansiedad y el cansancio que esta intervención podría producir, teniendo en cuenta el natural cansancio y estado anímico de la mujer en esta época y la repercusión en el vínculo madre e hijo.

Sabemos que el peso ganado durante el embarazo es un determinante en la obesidad. Clásicamente no se recomendaba ningún tipo de intervención para bajar de peso en esta etapa de la vida. Según este estudio parecería que intervenir con dieta y ejercicio, no implicaría riesgo para la salud del niño. Las preguntas pendientes son: ¿debemos intervenir en este momento?; ¿no deberíamos intervenir durante el embarazo, o luego de los 4 a 6 meses en que el niño comienza a ingerir otros alimentos?

\section{Dra. Claudia C. Dreyer}

Unidad de Medicina Familiar y Preventiva . Hospital Italiano de Buenos Aires.

\section{Referencias}

1. Flegal KM, Carroll MD, Kuczmarski RJ, Jhonson CL. Overweight and obesity in the United States: prevalence and trends, 1960-1994. Int J 0bes Relar Metab Disorde 1998; 22: 39-47.

2. Dusdiekere LB, Hemingway DL, Stumbo PJ. ¿Is milk production impaired by dieting during lactation?. Am J Clin Nutri 1994; 59: 833-40.

3. Jinich P, Barán P, .Algunos aspectos del control del niño en el primer año de vida: En: PROFAM: Programa de educación a distancia de medicina familiar y ambulatoria / Esteban Rubinstein, dir. Hospital Italiano de Buenos Aires: Departamento de Docencia e Investigación, 1999. v. 8, p. 77-115. 